\title{
DUALITY FOR LINEAR CHANCE-CONSTRAINED OPTIMIZATION PROBLEMS
}

\author{
Radu Ioan Bots, Nicole Lorenz, and Gert Wanka
}

\begin{abstract}
In this paper we deal with linear chance-constrained optimization problems, a class of problems which naturally arise in practical applications in finance, engineering, transportation and scheduling, where decisions are made in presence of uncertainty. After giving the deterministic equivalent formulation of a linear chance-constrained optimization problem we construct a conjugate dual problem to it. Then we provide for this primal-dual pair weak sufficient conditions which ensure strong duality. In this way we generalize some results recently given in the literature. We also apply the general duality scheme to a portfolio optimization problem, a fact that allows us to derive necessary and sufficient optimality conditions for it.
\end{abstract}

\section{Introduction}

Stochastic programming is an important topic in the optimization theory with applications in various fields like financial systems, engineering, location and transportation problems or the study of physical and chemical systems. The optimization problems occurring in all these fields usually contain uncertain variables either in the objective functions or in the constraints or even in both. The interest in optimization under uncertainty was revived in the 1950s and is still of importance. We refer for an overview on stochastic programming to $[16]$.

In this paper we work with stochastic programming problems with deterministic objective functions and chance (probabilistic)-constraints, a class of problems that has been first investigated by Charnes and Cooper in $[6,7,8]$. Other authors which dealt with such problems very early were van de Panne and Popp in [18] and Kataoka in [12]. The problem we consider in this article

Received December 10, 2007; Revised February 9, 2009

2000 Mathematics Subject Classification. 49N15, 90C46, 46N10.

Key words and phrases. stochastic programming, conjugate duality, optimality conditions, chance-constraints, portfolio optimization. 
is

$$
\begin{aligned}
\left(P_{\text {mix }}\right) \text { inf } & f(x), \\
\text { s.t. } & \mathbb{P}\left(g_{i}(x) \leq 0\right) \geq 1-\alpha_{i}, i=1, \ldots, m, \\
& h_{j}(x) \leq 0, j=1, \ldots, k, \\
& x \in \mathbb{R}^{n}
\end{aligned}
$$

where $f$ and $h_{j}, j=1, \ldots, k$, are convex functions while $g_{i}, i=1, \ldots, m$, are linear functions with random values. These constraints are called single chance-constraints since individual probabilities ensure that every inequality holds.

In many papers the use of the theory of chance-constraints in practical applications is considered and in this setting the case of the single chance-constraints play an important role. Single chance-constraints may be for instance used when some constraints are more critical then other ones. Practical applications using single chance-constraints have been given e.g. for portfolio optimization problems (cf. [4]), but also for problems in production, transport and operations planning.

The approach considered in this paper assumes giving a deterministic equivalent formulation of the linear single chance-constraints (see $[4,7,11]$ ). This transforms $\left(P_{\text {mix }}\right)$ into a convex deterministic optimization problem. To this aim one has to assume that the distribution of the uncertain variables is known. In this paper we even postulate a normal distribution, but in general also other distributions can be considered (cf. $[1,5,9]$ ). It is worth mentioning that in general the chance-constraints can be assumed to be also nonlinear, even if in this case the determining of their distribution functions could be a difficult task.

The paper is organized as follows. In the next section we introduce some definitions and notations from the convex analysis and employ some results from the stochastic theory in order to give an equivalent deterministic formulation to $\left(P_{\operatorname{mix}}\right)$. In Section 3 we construct a conjugate dual to $\left(P_{\operatorname{mix}}\right)$ and give a strong duality theorem along with a regularity condition which guarantees it. As a special case of $\left(P_{\text {mix }}\right)$ we consider first in Section 4 an optimization problem with linear objective function and linear (chance)-constraints and provide a dual problem to it. In this way we rediscover and extend a result recently given by Scott and Jefferson in [17], which turns out to be a special case of our considerations. As a practical application to financial mathematics we further provide the dual of a portfolio optimization problem with chance-constraints by means of the general duality scheme and derive necessary and sufficient optimality conditions for it. The last section gives some directions for future research. 


\section{Preliminary notions and results}

In this section we introduce some notations we use within the paper along with some well-known results. Throughout this paper all the vectors are considered as being column vectors. An upper index ${ }^{T}$ transposes a column vector into a row one and viceversa. For two arbitrary vectors $x, y \in \mathbb{R}^{n}$ the usual $i n$ ner product in the $n$-dimensional real space is denoted by $x^{T} y$. For $i=1, \ldots, n$ by $e_{i}$ we denote the $i$-th unit vector in $\mathbb{R}^{n}$ and by $e=(1, \ldots, 1)^{T} \in \mathbb{R}^{n}$.

The prefix ri is used for the relative interior of a set, while for the effective domain of a function $f: \mathbb{R}^{n} \rightarrow \overline{\mathbb{R}}:=\mathbb{R} \cup\{ \pm \infty\}$ we use the notation $\operatorname{dom}(f)=\left\{x \in \mathbb{R}^{n}: f(x)<+\infty\right\}$. The function $f$ is said to be proper if $\operatorname{dom}(f) \neq \emptyset$ and $f(x)>-\infty \forall x \in \mathbb{R}^{n}$. For $f: \mathbb{R}^{n} \rightarrow \overline{\mathbb{R}}$ we consider the conjugate function relative to the nonempty set $D \subseteq \mathbb{R}^{n}$ that is defined by $f_{D}^{*}\left(x^{*}\right)=\sup _{x \in D}\left\{x^{T} x^{*}-f(x)\right\}$. Obviously, for $D=\mathbb{R}^{n}, f_{D}^{*}$ becomes the (Fenchel-Moreau) conjugate function of $f$, which is denoted by $f^{*}$. For an optimization problem $(P)$ we denote by $v(P)$ its optimal objective value. We write $\min (\max )$ instead of inf ( $\sup )$ if the infimum (supremum) is attained.

Let $(\Omega, \mathfrak{F}, \mathbb{P})$ be a probability space, where $\Omega$ is a basic space, $\mathfrak{F}$ a $\sigma$-algebra on $\Omega$ and $\mathbb{P}$ a probability measure on the measurable space $(\Omega, \mathfrak{F})$. Furthermore, let $\mathbb{L}_{2}$ be the following space of random variables:

$$
\mathbb{L}_{2}:=\mathbb{L}_{2}(\Omega, \mathfrak{F}, \mathbb{P}, \mathbb{R})=\left\{x: \Omega \rightarrow \mathbb{R}, x \text { measurable, } \int_{\Omega} x(\omega)^{2} d \mathbb{P}(\omega)<+\infty\right\}
$$

By $\eta \sim \mathcal{N}(0,1)$ we denote a standard normal distributed random variable. For $z \in \mathbb{R}$ it holds $\mathbb{P}(\eta \leq z)=\Phi(z)$ and $\mathbb{P}(z \leq \eta)=1-\Phi(z)$, where $\Phi$ is the distribution function of the standard normal distribution. For $\theta \sim \mathcal{N}\left(\mu, \sigma^{2}\right)$ we have $\frac{\theta-\mu}{\sigma} \sim \mathcal{N}(0,1)$ and so

$$
\mathbb{P}(\theta \leq z)=\mathbb{P}\left(\frac{\theta-\mu}{\sigma} \leq \frac{z-\mu}{\sigma}\right)=\Phi\left(\frac{z-\mu}{\sigma}\right) .
$$

Let $K_{\alpha}:=\Phi^{-1}(1-\alpha)$ be the $(1-\alpha)$-quantile of the standard normal distribution, which is a positive number when $\alpha \in(0,0.5]$.

For $x, y$ two random variables we denote by $\mathbb{E}(x)$ the expected value of $x$, by $\mathbb{D}^{2}(x)$ its variance and by $\operatorname{cov}(x, y)=\mathbb{E}((x-\mathbb{E}(x))(y-\mathbb{E}(y))$ the covariance between $x$ and $y$. Let $a_{j} \in \mathbb{L}_{2}, j=1, \ldots, n$ and $b \in \mathbb{L}_{2}$ be normal distributed random variables. We denote $a:=\left(a_{1}, \ldots, a_{n}\right)$ and introduce the notation $\mathbb{E}(a):=\left(\mathbb{E}\left(a_{1}\right), \ldots, \mathbb{E}\left(a_{n}\right)\right)^{T}$ for the vector having as components the expected value of the components of $a$. For $x \in \mathbb{R}^{n}$ let be $a^{T} x:=\sum_{j=1}^{n} a_{j} x_{j}$. The symmetric positive semidefinite so-called variance-covariance matrix of a will be denoted by

$$
\Sigma(a)=\left(\begin{array}{cccc}
\mathbb{D}^{2}\left(a_{1}\right) & \operatorname{cov}\left(a_{1}, a_{2}\right) & \ldots & \operatorname{cov}\left(a_{1}, a_{n}\right) \\
\operatorname{cov}\left(a_{2}, a_{1}\right) & \mathbb{D}^{2}\left(a_{2}\right) & \ldots & \operatorname{cov}\left(a_{2}, a_{n}\right) \\
\vdots & & \ddots & \vdots \\
\operatorname{cov}\left(a_{n}, a_{1}\right) & \ldots & \ldots & \mathbb{D}^{2}\left(a_{n}\right)
\end{array}\right) .
$$


Next we assume a multinormal distribution $a_{j}, j=1, \ldots, k$, and $b$ and define the function $g: \mathbb{R}^{n} \rightarrow \mathbb{L}_{2}$ by $g(x):=a^{T} x-b$. For $\alpha \in(0,0.5]$ we consider the following chance-constraint $\mathbb{P}(g(x) \leq 0) \geq 1-\alpha$. Since for $x \in \mathbb{R}^{n}$ $g(x)$ is a linear combination of normal distributed random variables, it has an (one-dimensional) joint normal distribution with the expected value $\mathbb{E}(g(x))=$ $\sum_{j=1}^{n} \mathbb{E}\left(a_{j}\right) x_{j}-\mathbb{E}(b)=x^{T} \mathbb{E}(a)-\mathbb{E}(b)$.

Let be further $C(a, b):=\left(\operatorname{cov}\left(a_{1}, b\right), \ldots, \operatorname{cov}\left(a_{n}, b\right)\right)^{T} \in \mathbb{R}^{n}$. The $n+1$-tuple $\left(a_{1}, \ldots, a_{n}, b\right)$ of random variables has a normal distribution and a symmetric positive semidefinite variance-covariance matrix

$$
S(a, b):=\left(\begin{array}{cc}
\Sigma(a) & C(a, b) \\
C(a, b)^{T} & \mathbb{D}^{2}(b)
\end{array}\right) \in \mathbb{R}^{(n+1) \times(n+1)} .
$$

Then $g(x)$ has as variance $\mathbb{D}^{2}(g(x)):=z(x)^{T} S(a, b) z(x)=x^{T} \Sigma x-2 C(a, b)^{T} x+$ $\mathbb{D}^{2}(b)$, where $z(x):=\left(x_{1}, \ldots, x_{n},-1\right)^{T} \in \mathbb{R}^{n+1}$, and, consequently, it holds $\mathbb{D}^{2}(g(x)) \geq 0$ for all $x \in \mathbb{R}^{n}$. Therefore the standard deviation of $g(x)$ is $\mathbb{D}(g(x))=\sqrt{z(x)^{T} S(a, b) z(x)}=\sqrt{x^{T} \Sigma(a) x-2 C^{T}(a, b) x+\mathbb{D}^{2}(b)}$.

As well-known from the literature the constraint $\mathbb{P}(g(x) \leq 0) \geq 1-\alpha$ has as equivalent deterministic formulation $\tilde{g}(x):=\mathbb{E}(g(x))+\mathbb{D}(\bar{g}(x)) \cdot K_{\alpha} \leq 0$. It also holds $\mathbb{P}(g(x) \leq 0)>1-\alpha \Leftrightarrow \mathbb{E}(g(x))+\mathbb{D}(g(x)) \cdot K_{\alpha}<0$. As mentioned above, for $\alpha \in(0,0.5]$ one has $K_{\alpha}>0$. In this situation the function $\tilde{g}$ turns out to be a convex function (cf. $[12,14,18]$ ).

Let us mention that one can also consider in this setting distributions in the chance-constraints that are different from the normal one, like the elliptical symmetric and the log-concave symmetric distributions (cf. [10, 13]). In this situations an equivalent deterministic reformulation of the chance-constraints is possible, too.

On can notice that the function $f: \mathbb{R}^{n} \rightarrow \mathbb{R}$

$$
f(x)=\sqrt{x^{T} \Sigma(a) x-2 C(a, b)^{T} x+\mathbb{D}^{2}(b)},
$$

with $a=\left(a_{1}, \ldots, a_{n}\right), b, \Sigma(a)$ and $C(a, b)$ as above, will play an important role in our investigations. The calculation of its conjugate function is not a trivial task and employs some knowledge from the convex duality theory. This leads for all $x^{*} \in \mathbb{R}^{n}$ to the following formula

$$
f^{*}\left(x^{*}\right)=\min _{\lambda_{2} \in \mathbb{R}}\left\{\lambda_{2}+s^{*}\left(x^{*}, \lambda_{2}\right)\right\},
$$

where by $s: \mathbb{R}^{n} \rightarrow \mathbb{R}$ we denote the function $s(x)=\sqrt{x^{T} S(a, b) x}$ for all $x \in \mathbb{R}^{n}$. For an arbitrary $\lambda \in \mathbb{R}^{n+1}$ we have that $s^{*}(\lambda)=0$ if there exists $w \in \mathbb{R}^{n+1}$ such that $\lambda=S(a, b) w$ and $w^{T} S(a, b) w \leq 1$ and $s^{*}(\lambda)=+\infty$ otherwise. This provides the following formula for the conjugate of $f$ for all $x^{*} \in \mathbb{R}^{n}$ :

$$
f^{*}\left(x^{*}\right)=\min _{\substack{u \in \mathbb{R}^{n}, v \in \mathbb{R}, x^{*}=\Sigma(a) u+C(a, b) v, u^{T} \Sigma(a) u+2 v C(a, b)^{T} u+v^{2} \mathbb{D}^{2}(b) \leq 1}}\left\{C(a, b)^{T} u+\mathbb{D}^{2}(b) v\right\} .
$$




\section{Duality for the chance-constrained problem}

In this section we develop a duality scheme for the optimization problem with mixed (chance- and convex) constraints:

$$
\begin{aligned}
\left(P_{\text {mix }}\right) \text { inf } & f(x) . \\
\text { s.t. } & \mathbb{P}\left(g_{i}(x) \leq 0\right) \geq 1-\alpha_{i}, i=1, \ldots, m \\
& h_{j}(x) \leq 0, j=1, \ldots, k \\
& x \in \mathbb{R}^{n}
\end{aligned}
$$

To this aim we assume that $f: \mathbb{R}^{n} \rightarrow \overline{\mathbb{R}}$ and $h_{j}: \mathbb{R}^{n} \rightarrow \mathbb{R}, j=1, \ldots, k$, are convex functions and that $g_{i}: \mathbb{R}^{n} \rightarrow \mathbb{L}_{2}$ are defined by $g_{i}(x)=\sum_{j=1}^{n} a_{i j} x_{j}-$ $b_{i}=a_{i}^{T} x-b_{i}$ for $i=1, \ldots, m$, . Here $a_{i j}, b_{i} \in \mathbb{L}_{2}, i=1, \ldots, m, j=1, \ldots, m$, are random variables, $\alpha_{i} \in(0,0.5]$ and $a_{i}:=\left(a_{i 1}, \ldots, a_{i n}\right)$ for $i=1, \ldots, m$.

The investigations done in this section concerning $\left(P_{\text {mix }}\right)$ extend the ones recently made by Scott and Jefferson in [17]. As a byproduct, by using the duality theory, we derive in the following section necessary and sufficient optimality conditions for the portfolio optimization problem with chance-constraints.

We start by noticing that, keeping the notation from the previous section, the problem $\left(P_{\text {mix }}\right)$ can be equivalently written as:

$$
\begin{aligned}
\left(P_{\text {mix }}\right) \text { inf } & f(x) . \\
\text { s.t. } \quad & x^{T} \mathbb{E}\left(a_{i}\right)-\mathbb{E}\left(b_{i}\right)+\sqrt{x^{T} \Sigma\left(a_{i}\right) x-2 C\left(a_{i}, b_{i}\right)^{T} x+\mathbb{D}^{2}\left(b^{i}\right)} \cdot K_{\alpha_{i}} \leq 0 \\
& i=1, \ldots, m \\
& h_{j}(x) \leq 0, j=1, \ldots, k \\
& x \in \mathbb{R}^{n}
\end{aligned}
$$

For further calculations we denote for $i=1, \ldots, m$ by $f_{i}: \mathbb{R}^{n} \rightarrow \mathbb{R}$ and $\tilde{g}_{i}$ : $\mathbb{R}^{n} \rightarrow \mathbb{R}$ the functions defined as $f_{i}(x)=\sqrt{x^{T} \Sigma\left(a_{i}, b_{i}\right) x-2 C\left(a_{i}, b_{i}\right)^{T} x+\mathbb{D}^{2}\left(b^{i}\right)}$ and $\tilde{g}_{i}(x)=\mathbb{E}\left(g_{i}(x)\right)+\mathbb{D}\left(g_{i}(x)\right) \cdot K_{\alpha_{i}}$, respectively. Let also be $\tilde{g}:=\left(\tilde{g}_{1}, \ldots, \tilde{g}_{m}\right)^{T}$ and $h:=\left(h_{1}, \ldots, h_{k}\right)^{T}$. First we construct the Lagrange dual problem to $\left(P_{\text {mix }}\right)$. This has the following formulation

$$
\left(D_{\text {mix }}\right) \quad \sup _{\beta \in \mathbb{R}_{+}^{m}, \gamma \in \mathbb{R}_{+}^{k}} \inf _{x \in \mathbb{R}^{n}}\left\{f(x)+\beta^{T} \tilde{g}(x)+\gamma^{T} h(x)\right\}
$$

or, equivalently,

$$
\left(D_{\text {mix }}\right) \quad \sup _{\beta \in \mathbb{R}_{+}^{m}, \gamma \in \mathbb{R}_{+}^{k}}\left\{-\left(f+\sum_{i=1}^{m} \beta_{i} K_{\alpha_{i}} f_{i}+\sum_{j=1}^{k} \gamma_{j} h_{j}\right)^{*}\left(-\sum_{i=1}^{m} \beta_{i} \mathbb{E}\left(a_{i}\right)\right)-\sum_{i=1}^{m} \beta_{i} \mathbb{E}\left(b_{i}\right)\right\} .
$$

For the conjugates of $f_{i}, i=1, \ldots, k$, one can use now the formula given in the previous section. On the other hand, the conjugate of the sum of convex functions can be written in this setting as being equal to the infimal convolution of the conjugates of the summands (cf. [15]). Consequently, after some 
calculations and transformations we obtain the following dual problem:

$$
\begin{aligned}
\left(D_{\text {mix }}\right) \sup _{\substack{\beta_{i} \in \mathbb{R}_{+}, u_{i} \in \mathbb{R}^{n}, v_{i} \in \mathbb{R}, u_{i}^{T} \Sigma\left(a_{i}\right) u_{i}+2 v_{i} C\left(a_{i}, b_{i}\right)^{T} u_{i} \\
+v_{i}^{2} \mathbb{D}^{2}\left(b_{i}\right) \leq 1, i=1, \ldots, m, \gamma_{j} \in \mathbb{R}_{+}, q_{j} \in \mathbb{R}^{n}, j=1, \ldots, k}} & \left\{-f^{*}\left(-\sum_{i=1}^{m} \beta_{i}\left[\mathbb{E}\left(a_{i}\right)+K_{\alpha_{i}}\left(\Sigma\left(a_{i}\right) u_{i}+C\left(a_{i}, b_{i}\right) v_{i}\right)\right]-\sum_{j=1}^{k} \gamma_{j} q_{j}\right)\right. \\
& \left.-\sum_{i=1}^{m} \beta_{i} \mathbb{E}\left(b_{i}\right)-\sum_{i=1}^{m} \beta_{i} K_{\alpha_{i}}\left[C\left(a_{i}, b_{i}\right)^{T} u_{i}+\mathbb{D}^{2}\left(b_{i}\right) v_{i}\right]-\sum_{j=1}^{k} \gamma_{j} h_{j}^{*}\left(q_{j}\right)\right\} .
\end{aligned}
$$

For having strong duality for the primal-dual pair $\left(P_{\text {mix }}\right)-\left(D_{\text {mix }}\right)$ one needs to have a constraint qualification fulfilled. In our situation it is enough to demand that (see [15])

$$
\left(C Q_{\text {mix }}\right) \quad \exists x^{\prime} \in \operatorname{ri}(\operatorname{dom}(f)):\left\{\begin{array}{l}
\tilde{g}_{i}\left(x^{\prime}\right)<0, i=1, \ldots, m \\
h_{j}\left(x^{\prime}\right) \leq 0, j \in L \\
h_{j}\left(x^{\prime}\right)<0, j \in N
\end{array}\right.
$$

is fulfilled, where $L=\left\{j \in\{1, \ldots, k\}: h_{j}\right.$ is affine $\}$ and $N=\{1, \ldots, k\} \backslash L$. It is easy to see that this constraint qualification can be equivalently written as

$$
\left(C Q_{\text {mix }}\right) \quad \exists x^{\prime} \in \operatorname{ri}(\operatorname{dom}(f)): \quad\left\{\begin{array}{l}
\mathbb{P}\left(g_{i}\left(x^{\prime}\right) \leq 0\right)>1-\alpha_{i}, i=1, \ldots, m, \\
h_{j}\left(x^{\prime}\right) \leq 0, j \in L, \\
h_{j}\left(x^{\prime}\right)<0, j \in N .
\end{array}\right.
$$

Now we can state the so-called strong duality theorem.

Theorem 3.1 (strong duality for $\left.\left(P_{\text {mix }}\right)-\left(D_{\text {mix }}\right)\right)$. Let us assume that $\left(C Q_{\text {mix }}\right)$ is fulfilled. Then it holds $v\left(P_{\text {mix }}\right)=v\left(D_{\text {mix }}\right)$ and the dual $\left(D_{\text {mix }}\right)$ has an optimal solution.

\section{Particular cases}

Within this section we show first how a recent result due to Scott and Jefferson follows as a particular case of our general duality scheme, while in the second part we derive necessary and sufficient optimality conditions for a portfolio optimization problem with linear chance-constraints.

\subsection{The linear case}

Consider the optimization problem

$$
\begin{aligned}
\left(P_{\text {lin }}\right) \text { inf } & a^{T} x, \\
\text { s.t. } \quad & \mathbb{P}\left(\sum_{j=1}^{n} a_{i j} x_{j} \leq b_{i}\right) \geq 1-\alpha_{i}, i=1, \ldots, m, \\
& x \geqq 0
\end{aligned}
$$

where $a \in \mathbb{R}^{n}$ and $a_{i j}, b_{i} \in \mathbb{L}_{2}, i=1, \ldots, m, j=1, \ldots, m$ are random variables, $\alpha_{i} \in(0,0.5]$ and $a_{i}:=\left(a_{i 1}, \ldots, a_{i n}\right)$ for $i=1, \ldots, m$. 
A dual problem to $\left(P_{\text {lin }}\right)$ will be constructed by applying the general theory in Section 3 , namely by particularizing $\left(D_{\text {mix }}\right)$. This will have the following formulation

$$
\begin{aligned}
& \left(D_{\text {lin }}\right) \quad \sup _{\substack{\beta_{i} \in \mathbb{R}_{+}, u_{i} \in \mathbb{R}^{n}, v_{i} \in \mathbb{R}, C\left(a_{i}, b_{i}\right)^{T} u_{i}+v_{i}^{2} \mathbb{D}^{2}\left(b_{i}\right) \leq 1,}}\left\{-\sum_{i=1}^{m} \beta_{i}\left[\mu_{b}^{i}+K_{\alpha_{i}}\left(C\left(a_{i}, b_{i}\right)^{T} u_{i}+\mathbb{D}^{2}\left(b_{i}\right) v_{i}\right)\right]\right\} \\
& u_{i}^{T} \Sigma\left(a_{i}\right) u_{i}+2 v^{2}, m, \\
& -\sum_{i=1}^{m} \beta_{i}\left[\mathbb{E}\left(a_{i}\right)+K_{\alpha_{i}}\left(\Sigma\left(a_{i}\right) u_{i}+C\left(a_{i}, b_{i}\right) v_{i}\right)\right] \\
& -\sum_{j=1}^{n} \gamma_{j} q_{j}=a, \gamma_{j} \in \mathbb{R}_{+}, q_{j}=-e_{j}, j=1, \ldots, n
\end{aligned}
$$

or, equivalently,

$$
\begin{gathered}
\left(D_{\text {lin }}\right) \underset{\substack{\beta_{i} \in \mathbb{R}_{+}, u_{i} \in \mathbb{R}^{n}, v_{i} \in \mathbb{R}, u_{i}^{T} \Sigma\left(a_{i} u_{i}+2 v_{i} C\left(a_{i}, b_{i}\right)^{T} u_{i}\right.}}{+v_{i}^{2} \mathbb{D}^{2}\left(b_{i}\right) \leq 1, i=1, \ldots, m,}\left(\sum_{i=1}^{m} \beta_{i}\left[\mathbb{E}\left(b_{i}\right)+K_{\alpha_{i}}\left(C\left(a_{i}, b_{i}\right)^{T} u_{i}+\mathbb{D}^{2}\left(b_{i}\right) v_{i}\right)\right]\right\} . \\
a+\sum_{i=1}^{m} \beta_{i}\left[\mathbb{E}\left(a_{i}\right)+K_{\alpha_{i}}\left(\Sigma\left(a_{i}\right) u_{i}+C\left(a_{i}, b_{i}\right) v_{i}\right)\right] \geq 0
\end{gathered}
$$

A particular instance of $\left(P_{\text {lin }}\right)$ was recently treated by Scott and Jefferson in [17] by means of geometric programming duality. The primal problem in the probabilistic formulation is given in the form of $\left(P_{\text {lin }}\right)$ while the deterministic equivalent formulation of it is

$$
\begin{aligned}
\left(P_{\text {lin, SJ }}\right) \text { inf } & a^{T} x . \\
\text { s.t. } & x^{T} \mathbb{E}\left(a^{i}\right)-b_{i}+K_{\alpha_{i}} \sqrt{x^{T} \Sigma\left(a_{i}\right) x} \leq 0, i=1, \ldots, k, \\
& x^{T} a_{i}-\mathbb{E}\left(b_{i}\right)+K_{\alpha_{i}} \mathbb{D}\left(b_{i}\right) \leq 0, i=k+1, \ldots, m, \\
& x \geqq 0
\end{aligned}
$$

In [17] the authors actually suppose that for $1<k<m, a_{i j}, j=1, \ldots, n$, are normal distributed for $i=1, \ldots, k$ and deterministic for $i=k+1, \ldots, m$, while $b_{i}$ are normal distributed for $i=k+1, \ldots, m$ and deterministic for $i=1, \ldots, k$. Further, $\Sigma\left(a_{i}\right)$ are assumed to be positive definite for $i=1, \ldots, k$. This implies that

$$
\left\{\begin{array}{l}
\mathbb{D}^{2}\left(b_{i}\right)=0 \quad \text { and } \quad \mathbb{E}\left(b_{i}\right)=b_{i}, \quad \forall i \leq k \\
\Sigma\left(a_{i}\right)=0 \quad \text { and } \mathbb{E}\left(a_{i}\right)=a_{i}, \forall i \geq k+1 \\
C\left(a_{i}, b_{i}\right)=0, \forall i=1, \ldots, m
\end{array}\right.
$$

The dual $\left(D_{\text {lin }}\right)$ provides under the usage of $(2)$ the following dual problem to $\left(P_{\text {lin, SJ }}\right)$

$$
\begin{gathered}
\left.\sup _{\text {lin }, \mathrm{SJ}}\right) \underset{\substack{\beta_{i} \in \mathbb{R}_{+}, u_{i} \in \mathbb{R}^{n}, u_{i}^{T} \Sigma\left(a_{i}\right) u_{i} \leq 1, i=1, \ldots, k, v_{i} \in \mathbb{R}, v_{i}^{2} \mathbb{D}^{2}\left(b_{i}\right) \leq 1, i=k+1, \ldots, m,}}{a+\sum_{i=1}^{k} \beta_{i}\left[\mathbb{E}\left(a_{i}\right)+K_{\alpha_{i}} \Sigma\left(a_{i}\right) u_{i}\right]+\sum_{i=k+1}^{m} \beta_{i} a_{i} \geq 0} \\
\end{gathered}
$$


Some further treatments (in particular introducing the new variables $p_{i}:=$ $-\beta_{i} K_{\alpha_{i}} \Sigma_{i} u_{i}$ for $\left.i=1, \ldots, k\right)$ yield the following formulation of the dual problem to $\left(P_{\text {lin, SJ }}\right)$

$$
\left(D_{\text {lin }, \mathrm{SJ}}\right) \underset{\substack{\beta_{i} \in \mathbb{R}_{+}, p_{i} \in \mathbb{R}^{n}, \sqrt{p_{i}^{T} \Sigma\left(a_{i}\right)^{-1} p_{i}} \leq \beta_{i} K_{\alpha_{i}} \\ i=1, \ldots, k, a+\sum_{i=1}^{k} \beta_{i} \mathbb{E}\left(a_{i}\right)-\sum_{i=1}^{k} p_{i}+\sum_{i=k+1}^{m} \beta_{i} a_{i} \geq 0}}{ }\left\{-\sum_{i=1}^{k} \beta_{i} b_{i}-\sum_{i=k+1}^{m} \beta_{i}\left[\mathbb{E}\left(b^{i}\right)-K_{\alpha_{i}} \mathbb{D}\left(b_{i}\right)\right]\right\} .
$$

The constraint qualification given in Section 3 becomes in this special case:

$$
\left(C Q_{\text {lin, SJ }}\right) \exists x^{\prime} \in \mathbb{R}_{+}^{n}:\left\{\begin{array}{l}
\mathbb{P}\left(a_{i}^{T} x^{\prime}-b_{i} \leq 0\right)>1-\alpha_{i}, \quad i=1, \ldots, k, \\
\mathbb{P}\left(a_{i}^{T} x^{\prime}-b_{i} \leq 0\right) \geq 1-\alpha_{i}, \quad i=k+1, \ldots, m .
\end{array}\right.
$$

Theorem 4.1 (strong duality $\left.\left(P_{\text {lin,SJ }}\right)-\left(D_{\text {lin,SJ }}\right)\right)$. Let us assume that $\left(C Q_{\text {lin,SJ }}\right)$ is fulfilled. Then it holds $v\left(P_{\text {lin,SJ }}\right)=v\left(D_{\text {lin,SJ }}\right)$ and $\left(D_{\text {lin,SJ }}\right)$ has an optimal solution.

Remark 4.1. With the exception of a sign in the objective function, Scott and Jefferson give in [17] for $\left(P_{\text {lin,SJ }}\right)$ the same dual optimization problem as $\left(D_{\text {lin,SJ }}\right)$. Nevertheless, in that paper the problem of giving sufficient regularity conditions or of stating a strong duality theorem is not addressed.

\subsection{Application to portfolio optimization theory}

In this part we consider a portfolio optimization problem for which we derive necessary and sufficient optimality conditions as an application of the general duality scheme developed in Section 3. We assume that $n+1$ assets are given, where the first one is riskless with the riskless return $r_{0}=\mathbb{E}\left(r_{0}\right)$. The return of asset $j$ is given by the random variable $r_{j} \in \mathbb{L}_{2}$ with an expected return of $\mathbb{E}\left(r_{j}\right)$ for $j=1, \ldots, n$. We denote by $\mathbb{E}(r)$ the vector $\mathbb{E}(r):=$ $\left(\mathbb{E}\left(r_{0}\right), \mathbb{E}\left(r_{1}\right), \ldots, \mathbb{E}\left(r_{n}\right)\right)^{T}$. Further let $\Sigma(r) \in \mathbb{R}^{(n+1) \times(n+1)}$ be the symmetric positive semidefinite variance-covariance matrix of $r:=\left(r_{0}, r_{1}, \ldots, r_{n}\right)^{T}$. The classical portfolio optimization problem has the following formulation

$$
\begin{aligned}
\left(P_{\mathrm{po}}\right) \text { inf } & f(x) . \\
\text { s.t. } & x_{0} \mathbb{E}\left(r_{0}\right)+\sum_{j=1}^{n} x_{j} \mathbb{E}\left(r_{j}\right) \geq b, \\
& \sum_{j=0}^{n} x_{j}=1, \\
& x=\left(x_{0}, x_{1}, \ldots, x_{n}\right)^{T} \geqq 0
\end{aligned}
$$

where $x=\left(x_{0}, x_{1}, \ldots, x_{n}\right)^{T}$ is the vector having as components the proportions of the assets in the whole portfolio, $f: \mathbb{R}^{n} \rightarrow \mathbb{R}$ is a function measuring the risk of the portfolio (e.g. the variance or any deviation or risk measure). Here $b \in \mathbb{R}$ is a return benchmark and in our approach should be assumed to be a constant random variable in $\mathbb{L}_{2}$. Let $g: \mathbb{R}^{n+1} \rightarrow \mathbb{L}_{2}$ be defined by $g(x)=b-\sum_{j=0}^{n} x_{j} r_{j}=b-x^{T} r$. We have $\mathbb{E}(g(x))=b-x^{T} \mathbb{E}(r)$ and $\mathbb{D}^{2}(g(x))=$ 
$x^{T} \Sigma(r) x$ for all $x \in \mathbb{R}^{n+1}$. When applying the previous model to this particular situations one has to take $C(r, b)=0$ and $\mathbb{D}(b)=0$.

Remark 4.2. As proved in the previous section, one can construct a dual problem and also provide necessary and sufficient optimality conditions even if it considers that $b$ is a random variable in $\mathbb{L}_{2}$. This is of importance if our target is to construct a portfolio that hits or at least reaches any benchmark portfolio or market index (e.g. the DAX (Deutscher Aktienindex)) with a certain probability. Therefore one can consider $b=\sum_{j=1}^{n} y_{j} r_{j}$ with given and fixed proportions $y_{j} \in(0,1), j=1, \ldots, n$, and random returns $r_{j}, j=1, \ldots, n$, as above.

The following portfolio optimization problem with mixed (linear and chance-) constraints was treated in [4]

$$
\begin{aligned}
\left(P_{\text {pomix }}\right) \inf & f(x), \\
\text { s.t. } & \mathbb{P}\left(x_{0} r_{0}+\sum_{j=1}^{n} x_{j} r_{j} \geq b\right) \geq 1-\alpha, \\
& \sum_{j=0}^{n} x_{j}=1, \\
& x=\left(x_{0}, x_{1}, \ldots, x_{n}\right)^{T} \geqq 0
\end{aligned}
$$

where for $f$ the variance of the portfolio return was considered. Here we let $f$ being the standard deviation $f(x)=\sqrt{x^{T} \Sigma(r) x}, x \in \mathbb{R}^{n+1}$. Its equivalent deterministic formulation is

$$
\begin{aligned}
\left(P_{\text {pomix }}\right) \text { inf } & f(x), \\
\text { s.t. } & b-x^{T} \mathbb{E}(r)+K_{\alpha} \sqrt{x^{T} \Sigma(r) x} \leq 0, \\
& \sum_{j=0}^{n} x_{j}=1, \\
& x=\left(x_{0}, x_{1}, \ldots, x_{n}\right)^{T} \geqq 0
\end{aligned}
$$

By means of the general considerations made in Section 3 we obtain the following dual problem to $\left(P_{\text {pomix }}\right)$

$$
\begin{aligned}
& \sup _{\substack{\beta \in \mathbb{R}_{+}, u, w \in \mathbb{R}^{n+1}, u^{T} \Sigma(r) u \leq 1, w^{T} \Sigma(r) w \leq 1, \gamma_{j} \in \mathbb{R}_{+}, j=0, \ldots, n+2,}} \quad\left\{\beta b-\gamma_{n+1}+\gamma_{n+2}\right\} . \\
& -\beta\left(-\mathbb{E}(r)+K_{\alpha} \Sigma(r) u\right)+\sum_{j=0}^{n} \gamma_{j} e_{j}+e\left(-\gamma_{n+1}+\gamma_{n+2}\right)=\Sigma(r) w
\end{aligned}
$$

Further we define $\kappa:=\left(\gamma_{0}, \ldots, \gamma_{n}\right)^{T} \in \mathbb{R}_{+}^{n+1}$ and $c:=\gamma_{n+1}-\gamma_{n+2} \in \mathbb{R}$ and get

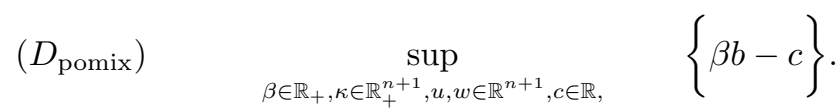

$$
\begin{aligned}
& u^{T} \Sigma(r) u<1, \quad w^{T} \Sigma(r) w \leq 1, \\
& c=\left[\kappa-\beta\left(-\mu+K_{\alpha} \Sigma(r) u\right)-\Sigma(r) w\right]_{j}, \forall j=0, \ldots, n
\end{aligned}
$$


The following constraint qualification

$$
\left(C Q_{\text {pomix }}\right) \quad \exists x^{\prime} \in \mathbb{R}_{+}^{n+1}:\left\{\begin{array}{l}
\mathbb{P}\left(b-\sum_{j=0}^{n} x_{j}^{\prime} r_{j} \leq 0\right)>1-\alpha, \\
\sum_{j=0}^{n} x_{j}^{\prime}=1
\end{array}\right.
$$

ensures for the primal-dual pair $\left(P_{\text {pomix }}\right)-\left(D_{\text {pomix }}\right)$ the existence of strong duality.

Theorem 4.2 (strong duality for $\left.\left(P_{\text {pomix }}\right)-\left(D_{\text {pomix }}\right)\right)$. Let us assume that $\left(C Q_{\text {pomix }}\right)$ is fulfilled. Then it holds $v\left(P_{\text {pomix }}\right)=v\left(D_{\text {pomix }}\right)$ and $\left(D_{\text {pomix }}\right)$ has an optimal solution.

The previous theorem is an important tool for deriving necessary and sufficient optimality conditions for the primal-dual pair $\left(P_{\text {pomix }}\right)-\left(D_{\text {pomix }}\right)$.

Theorem 4.3. (a) Let $\bar{x}$ be an optimal solution of ( $\left.P_{\text {pomix }}\right)$ and assume that $\left(C Q_{\text {pomix }}\right)$ is fulfilled. Then there exist $\bar{\beta} \geq 0, \bar{\kappa}=\left(\bar{\kappa}_{0}, \ldots, \bar{\kappa}_{n}\right)^{T}, \bar{\kappa}_{i} \geq 0, i=$ $0, \ldots, n, \bar{u}, \bar{w} \in \mathbb{R}^{n+1}$ with $\bar{u}^{T} \Sigma(r) \bar{u} \leq 1, \bar{w}^{T} \Sigma(r) \bar{w} \leq 1$ and $\bar{c} \in \mathbb{R}$ with $\bar{c}=\left[\bar{\kappa}-\bar{\beta}\left(-\mathbb{E}(r)+K_{\alpha} \Sigma(r) \bar{u}\right)-\Sigma(r) \bar{w}\right]_{j}, j=0, \ldots, n$, such that the following optimality conditions are fulfilled:

(i) $\sqrt{\bar{x}^{T} \Sigma(r) \bar{x}}\left[1-\sqrt{\bar{w}^{T} \Sigma(r) \bar{w}}\right]=0$,

(ii) $\sqrt{\bar{x}^{T} \Sigma(r) \bar{x}} \sqrt{\bar{w}^{T} \Sigma(r) \bar{w}}-\bar{x}^{T} \Sigma(r) \bar{w}=0$,

(iii) $\bar{\beta}\left[b-\bar{x}^{T} \mathbb{E}(r)+K_{\alpha} \sqrt{\bar{x}^{T} \Sigma(r) \bar{x}}\right]=0$,

(iv) $\bar{x}_{j} \bar{\kappa}_{j}=0, \quad j=0, \ldots, n$,

(v) $\bar{\beta} \sqrt{\bar{x}^{T} \Sigma(r) \bar{x}}\left(1-\sqrt{\bar{u}^{T} \Sigma(r) \bar{u}}\right)=0$,

(vi) $\bar{\beta}\left[\sqrt{\bar{x}^{T} \Sigma(r) \bar{x}} \sqrt{\bar{u}^{T} \Sigma(r) \bar{u}}-\bar{x}^{T} \Sigma(r) \bar{u}\right]=0$.

(b) If $\bar{x}$ is feasible to $\left(P_{\text {pomix }}\right)$ and $(\bar{\beta}, \bar{\kappa}, \bar{u}, \bar{w}, \bar{c})$ is feasible to $\left(D_{\text {pomix }}\right)$ fulfilling the optimality conditions (i)-(vi), then $\bar{x}$ is an optimal solution of $\left(P_{\text {pomix }}\right)$ and $(\bar{\beta}, \bar{\kappa}, \bar{u}, \bar{w}, \bar{c})$ is an optimal solution of $\left(D_{\text {pomix }}\right)$ and $v\left(P_{\text {pomix }}\right)=v\left(D_{\text {pomix }}\right)$.

Proof. (a) Since $\bar{x}$ is an optimal solution to $\left(P_{\text {pomix }}\right)$, by Theorem 4.2 there exists $(\bar{\beta}, \bar{\kappa}, \bar{u}, \bar{w}, \bar{c})$, an optimal solution to $\left(D_{\text {pomix }}\right)$, such that $v\left(P_{\text {pomix }}\right)=$ $v\left(D_{\text {pomix }}\right)$ or, equivalently, $\sqrt{\bar{x}^{T} \Sigma(r) \bar{x}}-\bar{\beta} b+\bar{c}=0$. But this is nothing else than

$$
\begin{aligned}
& {\left[\sqrt{\bar{x}^{T} \Sigma(r) \bar{x}}-\sqrt{\bar{x}^{T} \Sigma(r) \bar{x}} \sqrt{\bar{w}^{T} \Sigma(r) \bar{w}}\right]+\left[\sqrt{\bar{x}^{T} \Sigma(r) \bar{x}} \sqrt{\bar{w}^{T} \Sigma(r) \bar{w}}-\bar{x}^{T} \Sigma(r) \bar{w}\right]+\left[\bar{x}^{T} \bar{\kappa}\right]} \\
& +\left[-\bar{\beta}\left(b-\bar{x}^{T} \mathbb{E}(r)+K_{\alpha} \sqrt{\bar{x}^{T} \Sigma(r) \bar{x}}\right)\right]+\left[\bar{\beta} K_{\alpha}\left(\sqrt{\bar{x}^{T} \Sigma(r) \bar{x}} \sqrt{\bar{u}^{T} \Sigma(r) \bar{u}}-\bar{x}^{T} \Sigma(r) \bar{u}\right)\right] \\
& +\left[\bar{\beta} K_{\alpha}\left(\sqrt{\bar{x}^{T} \Sigma(r) \bar{x}}-\sqrt{\bar{x}^{T} \Sigma(r) \bar{x}} \sqrt{\left.\bar{u}^{T} \Sigma(r) \bar{u}\right)}\right]=0 .\right.
\end{aligned}
$$

All the terms inside the brackets are nonnegative and thus all of them have to be equal to zero and the conclusion follows. 
(b) The calculations done within part (a) can be carried out in reverse direction and therefore the proof is complete.

Remark 4.3. When $\Sigma(r)$ is a symmetric and positive definite matrix the conditions (i)-(vi) in the previous theorem can be written as follows (since one has $\left.\bar{x}^{T} \Sigma(r) \bar{x}>0\right)$ :
(i) $\bar{w}^{T} \Sigma(r) \bar{w}=1$,
(ii) $\sqrt{\bar{x}^{T} \Sigma(r) \bar{x}}-\bar{x}^{T} \Sigma(r) \bar{w}=0$,
(iii) $\bar{\beta}\left[b-\bar{x}^{T} \mathbb{E}(r)+K_{\alpha} \sqrt{\bar{x}^{T} \Sigma(r) \bar{x}}\right]=0$,
(iv) $\bar{x}_{j} \bar{\kappa}_{j}=0, \quad j=0, \ldots, n$,
(v) $\bar{\beta}\left(1-\sqrt{\bar{u}^{T} \Sigma(r) \bar{u}}\right)=0$,
(vi) $\bar{\beta}\left[\sqrt{\bar{x}^{T} \Sigma(r) \bar{x}}-\bar{x}^{T} \Sigma(r) \bar{u}\right]=0$.

Remark 4.4. One can consider in the objective function of $\left(P_{\text {pomix }}\right)$ also other convex risk or deviation measures, find a dual to it and derive necessary and sufficient optimality conditions. To this end one needs to find the conjugate of the objective function. In [3], by using the conjugate duality, we have provided formulae for the conjugate functions of different risk and deviation measures used in the literature on mathematics of finance and the theory of risk.

\section{Future research}

In this section we discuss some directions for future research in duality for optimization problems with chance constraints. First, we want to mention again that it is possible to consider in the chance-constraints also other distributions than the normal one, such as the elliptical symmetric and log-concave symmetric distribution (see $[10,13]$ ). As one can see in [10] it is possible to get explicit deterministic equivalent formulations for the optimization problems even if for the random variables these distributions are supposed. On could try to provide a duality scheme for problems with chance constraints of the type $\mathbb{P}(g(x) \leq 0) \geq 1-\alpha$, where $g$ is linear, but also nonlinear, under the usage of one of the mentioned distributions.

In this paper we deal with the case of $\alpha \in(0,0.5]$ which causes $K_{\alpha}>0$ and hence we get that $\tilde{g}(x)=\mathbb{E}(g(x))+\mathbb{D}(g(x)) \cdot K_{\alpha}, x \in \mathbb{R}^{n}$, is a convex function. The case where $K_{\alpha}<0$ leads to a function $\tilde{g}$ which is the difference of two convex functions $\mathbb{E}(g(\cdot))$ and $\mathbb{D}(g(\cdot))$. Therefore one could try to use techniques coming from dc programming techniques (cf. [2]) in order to deal with duality in this case.

\section{References}

[1] F. M. Allen, R. N. Braswell, and P. V. Rao, Distribution-free approximations for chance constraints, Oper. Res. 22 (1974), no. 3, 610-621.

[2] R. I. Bot, I. B. Hodrea, and G. Wanka, Some new Farkas-type results for inequality systems with DC functions, J. Global Optim. 39 (2007), no. 4, 595-608.

[3] R. I. Boț, N. Lorenz, and G. Wanka, Dual Representations for Convex Risk Measures via Conjugate Duality, to appear in Journal of Optimization Theory and Applications, 2009, DOI: 10.1007/s10957-009-9595-3. 
[4] P. Bonami and M. A. Lejeune, An exact solution approach for portfolio optimization problems under stochastic and integer constraints, http://www.optimization-online.org, 2007.

[5] G. Calafiore and L. El Ghaouni, On distributionally robust chance-constrained linear programs, J. Optim. Theory Appl. 130 (2006), no. 1, 1-22.

[6] A. Charnes and W. W. Cooper, Chance-constrained programming, Management Sci. 6 (1959/1960), 73-79.

[7] _ _ Deterministic equivalents for optimizing and satisficing under chance constraints, Oper. Res. 11 (1963), 18-39.

[8] A. Charnes, W. W. Cooper, and G. H. Symonds, Cost horizons and certainty equivalents: An approach to stochastic programming of heating oil, Management Sci. 4 (1958), no. 3, 235-263.

[9] W. K. K. Haneveld and M. H. van der Vlerk, Integrated chance constraints: reduced forms and an algorithm, Comput. Manag. Sci. 3 (2006), no. 4, 245-269.

[10] R. Henrion, Structural properties of linear probabilistic constraints, Optimization 56 (2007), no. 4, 425-440.

[11] P. Kall and S. W. Wallace, Stochastic programming, John Wiley \& Sons, 1994.

[12] S. Kataoka, A stochastic programming model, Econometrica 31 (1963), 181-196.

[13] C. M. Lagoa, X. Li, and M. Sznaier, Probabilistically constrained linear programs and risk-adjusted controller design, SIAM J. Optim. 15 (2005), no. 3, 938-951.

[14] A. Prékopa, Stochastic Programming, Kluwer Academic Publishers, 1995.

[15] R. T. Rockafellar, Convex Analysis, Princeton University Press, 1970.

[16] A. Ruszczynski and A. Shapiro (Eds.), Stochastic Programming, Handbooks in Operations Research and Management Science, Vol. 10, Elsevier, Amsterdam, 2003.

[17] C. H. Scott and T. R. Jefferson, On duality for square root convex programs, Math. Methods Oper. Res. 65 (2007), no. 1, 75-84.

[18] C. van de Panne and W. Popp, Minimum-cost cattle feed under probabilistic protein constraints, Management Sci. 9 (1963), no. 3, 405-430.

RADU IOAN BOŢ

Chemnitz University of Technology

FACULTY OF MATHEMATICS

D-09107 Chemnitz, Germany

E-mail address: radu.bot@mathematik.tu-chemnitz.de

NiCOLE LORENZ

Chemnitz University of Technology

FACUlty OF MAThEMATiCS

D-09107 Chemnitz, Germany

E-mail address: nicole.lorenz@mathematik.tu-chemnitz.de

Gert Wanka

Chemnitz University of Technology

FACUlty of Mathematics

D-09107 Chemnitz, Germany

E-mail address: gert.wanka@mathematik.tu-chemnitz.de 